Revue

de I'histoire des religions
Revue de l'histoire des religions

$4 \mid 2013$

Sermo mysticus Mystique et langage entre Moyen Âge et époque moderne

\title{
La théologie mystique selon Giovanni Bona et ses sources médiévales
}

Mystical theology according to Giovanni Bona and its medieval sources

\section{Simon Icard}

\section{OpenEdition}

\section{Journals}

Édition électronique

URL : http://journals.openedition.org/rhr/8184

DOI : $10.4000 /$ rhr.8184

ISSN : 2105-2573

Éditeur

Armand Colin

Édition imprimée

Date de publication : 1 décembre 2013

Pagination : 653-666

ISBN : $978-2200928667$

ISSN : 0035-1423

Référence électronique

Simon Icard, «La théologie mystique selon Giovanni Bona et ses sources médiévales », Revue de

I'histoire des religions [En ligne], 4 | 2013, mis en ligne le 01 décembre 2016, consulté le 19 avril 2019

URL : http://journals.openedition.org/rhr/8184 ; DOI : 10.4000/rhr.8184 


\section{SIMON ICARD}

Laboratoire d'études sur les monothéismes (LEM)

Centre national de la recherche scientifique

\section{La théologie mystique selon Giovanni Bona et ses sources médiévales}

L'ouvrage de Giovanni Bona Via compendii ad Deum (1657) est un traité des aspirations, chacune conçue comme un degré de l'ascension vers l'union mystique. Bona cite de nombreuses autorités, parmi lesquelles beaucoup d'auteurs médiévaux, car il entend présenter la plus constante tradition des doctrines spirituelles. Sa lecture du corpus médiéval est très importante dans l'élaboration de sa synthèse moderne sur la voie mystique.

Mystical theology according to Giovanni Bona and its medieval sources

Giovanni Bona's Via compendii ad Deum (1657) is a treatise on short prayers, each envisioned as a stage in the ascension towards mystical union. Bona cites many authorities, among them numerous medieval authors, because he intends to present the most constant tradition of spiritual doctrines. His interpretation of the medieval corpus is very important in his modern synthesis on the mystical pathway. 
Le $\mathrm{XVII}^{\mathrm{e}}$ siècle n'a pas prétendu apporter quoi que ce soit de nouveau en matière de spiritualité sans l'inscrire dans une tradition ininterrompue dont il se voulait solidaire. Et nous devons en particulier tenir compte du splendide héritage des médiévaux, trop souvent méconnu ou occulté, ne serait-ce que pour mieux comprendre les synthèses originales auxquelles le XVII ${ }^{\mathrm{e}}$ siècle a pu parvenir ${ }^{1}$.

Pour mesurer l'extrême pertinence de cette affirmation de Christian Belin, il suffit d'ouvrir un livre de spiritualité de Giovanni Bona (1609-1674). Cistercien de la congrégation italienne des feuillants, dont il est le général de 1651 à 1654 et de 1657 à 1664 , consulteur de plusieurs congrégations à Rome (Rites, Propagande, Saint-Office, Index), créé cardinal en 1669, Bona fut aussi un immense érudit. Lorsqu'il traite d'un sujet, que ce soit en matière de liturgie ou de spiritualité, il cherche à proposer une synthèse des autorités principales. Joseph-Marie Canivez notait ainsi sa grande connaissance des Pères de l'Église, mais aussi sa fréquentation des modernes, «particulièrement saint François de Sales, dont il a examiné les écrits en vue du procès de béatification, et saint Ignace de Loyola $»^{2}$. Assurément, il convient d'ajouter à cette liste les auteurs du Moyen Âge, dont il fut un lecteur assidu.

Dans son traité Via compendii ad Deum ${ }^{3}$, quelles sont les sources médiévales de Bona? Les auteurs du Moyen Âge les plus

1. Christian Belin, La Conversation intérieure. La méditation en France au XVII siècle, Paris, Honoré Champion, 2002, p. 366.

2. Joseph-Marie Canivez, «Bona (Jean)», Dictionnaire de spiritualité, Paris, Beauchesne, t. I, 1937, col. 1763.

3. Giovanni Bona, Via compendii ad Deum, per motus anagogicos, et orationes jaculatorias. Liber isagogicus ad mysticam theologiam, Rome, A. Bernabò a Verme, 1657. L'ouvrage a connu de très nombreuses rééditions au XVII ${ }^{\mathrm{e}}$ et au XVIII ${ }^{\mathrm{e}}$ siècles. Extrait du traité, le recueil des aspirations rédigées par Bona a connu également une carrière éditoriale importante, qui va de la fin du XVII ${ }^{\mathrm{e}}$ siècle à la fin du XIX ${ }^{\mathrm{e}}$ siècle. Je cite la traduction parue l'année suivant la mort de Bona: Voie abrégée pour aller à Dieu, Bruxelles, F. Foppens, 1675, en indiquant le latin en note lorsque j'ai modifié cette traduction ou qu'une précision est nécessaire. Sabrina Stroppa a publié récemment une édition bilingue latin / italien, avec introduction et notes: Via Compendii ad Deum /Via breve a Dio, Florence, L. S. Olschki, 2006; elle a étudié ce traité de Bona dans son ouvrage Sic arescit. Letteratura mistica del Seicento italiano, Florence, Olschki, 1998, parties I et III, et dans son article «L' "ars meditandi" nel seicento mistico», Rivista di Storia et Letteratura Religiosa, 2005-3, p. 515-536. Roberto Osculati lui consacre également un passage de son article «Vrai et faux christianisme dans l'œuvre théologique du cardinal Giovanni Bona (1609-1674)» 
cités dans ce livre sont Denys le chartreux, Jean Tauler, Richard de Saint-Victor, Bonaventure - ces deux derniers auteurs surtout sur la question de la contemplation - et Bernard de Clairvaux, évidemment serait-on tenté de dire, lui dont l'autorité est grande au $\mathrm{XVII}^{\mathrm{e}}$ siècle $^{4}$ et pour qui le feuillant avait une dévotion aussi forte que naturelle - «grand saint, je vous ai suivi par-dessus tout comme mon maître » déclare-t-il dans l'épître dédicatoire à l'abbé de Clairvaux qui ouvre son traité De discretione spirituum ${ }^{5}$. Bona cite également Guillaume de Saint-Thierry, Aelred de Rievaulx, Gilbert de Hoyland, Thomas d'Aquin, Angèle de Foligno, Jean de Ruysbroeck, Catherine de Sienne, Jean Gerson, Henri Harphius, Laurent Justinien, Thomas a Kempis et Catherine de Gênes.

Outre la méthode suivie par Bona, consistant à inscrire son propos dans la plus constante tradition des doctrines spirituelles, le sujet même de l'ouvrage rendait presque incontournables certains auteurs médiévaux. En effet, il s'agit d'un traité des aspirations. Bona les définit comme des «oraisons très courtes, ou seulement conçues par la pensée, ou même proférées de bouche ${ }^{6}$, auxquelles l'âme fidèle se doit accoutumer en tout temps et en tout lieu, dressant son cœur et sa volonté vers Dieu le jour et la nuit, à la maison ou dehors, assis ou debout, en quelque affaire, action et occupation que ce puisse être $»^{7}$. Il les appelle également «mouvements anagogiques, c'està-dire qui conduisent en haut», «oraisons jaculatoires, d'autant que comme des flèches très vites, on les tire au cour de Dieu comme au but, pour obtenir de lui des grâces célestes $»^{8}$ ou encore «affects [...] car ce sont des affections du cœur, des désirs et des intentions de la volonté ${ }^{9}$. Cette multiplication de termes censés

dans Les Piétismes à l'âge classique. Crise, conversion, institutions, dir. Anne Lagny, Villeneuve d'Ascq, Presses universitaires du Septentrion, 2001, p. 297-307.

4. Pour le contexte français, voir mon étude Port-Royal et saint Bernard de Clairvaux (1608-1709). Saint-Cyran, Jansénius, Arnauld, Pascal, Nicole, Angélique de Saint-Jean, Paris, Honoré Champion, 2010.

5. G. Bona, De discretione spirituum, Bruxelles, 1671. Sur ce traité, voir Simon Icard, «La tradition du discernement dans le traité De discretione spirituum de Jean Bona", dans Le Discernement spirituel au XVII siècle, dir. S. Icard, Paris, Nolin, 2011, p. 69-79.

6. «Sive mente simul ac corde conceptæ, atque prolatæ».

7. G. Bona, Voie abrégée pour aller à Dieu, p. 46.

8. Ibid., p. 48.

9. «Affectus denique appellantur, quia sunt affectiones cordis, et desideria, ac proposita voluntatis». Ibid., p. 48-49. 
être équivalents alors qu'ils ne sont pas vraiment synonymes ${ }^{10}$ est révélatrice du projet de l'ouvrage. Il s'agit de théoriser la pratique ancienne de la prière brève et spontanée, qui plonge ses racines dans la Bible, les Pères du désert et les textes fondateurs du monachisme ${ }^{11}$, pour la penser comme une «voie pour aller à Dieu», un itinéraire vers la contemplation et l'union mystique. Dans cette élaboration, les médiévaux jouent un rôle important, Bona héritant de leurs catégories et les modifiant en partie.

\section{LA THÉOLOGIE MYSTIQUE : UNE AUTHENTIQUE THÉOLOGIE}

Bona conclut son traité De discretione spirituum par une apologie de la théologie mystique et annonce une possible publication de sa part sur le sujet: "Il faudrait un volume entier pour défendre la théologie mystique contre les erreurs des ignorants: et nous en traiterons peut-être ailleurs Dieu aidant. ${{ }^{12}}^{12}$ Le traité Via compendii ad Deum, qui se présente comme une «introduction à la théologie mystique $»^{13}$, est-il cet ouvrage que Bona désirait écrire? La chronologie de ses œuvres ne permet pas de l'établir avec certitude. En effet, publié seulement en 1657, le traité Via compendii ad Deum était terminé dès $1650^{14}$. Le traité De discretione spirituum, quant à lui, fut rédigé durant l'abbatiat de Bona au monastère de Mondovi, qui s'achève en 1651 lorsqu'il devient général de sa congrégation, mais il fut repris et transformé au début des années $1670^{15}$. Quoi qu'il en soit, défendre la théologie mystique est une démarche commune aux deux ouvrages. Bona cherche à l'établir comme une authentique théologie, c'est-à-dire une connaissance de Dieu, supposant un discours sur Dieu. Dans la conclusion du De discretione spirituum, il affirme surtout la nécessité pour

10. Voir Edmond Vansteenberghe, «Aspirations », Dictionnaire de spiritualité, Paris, Beauchesne, t. I, 1937, col. 1018. Cet article doit visiblement beaucoup au traité de Bona.

11. Voir notamment Jean Cassien, $9^{\mathrm{e}}$ Conférence, $\S 36$ et Benoît de Nursie, Règle, ch. 20.

12. G. Bona, Traité du discernement des esprits, trad. du De discretione spirituum par Guillaume Le Roy, Paris, L. Billaine, 1675, p. 535.

13. Il porte le sous-titre de liber isagogicus ad theologiam mysticam.

14. Voir J.-M. Canivez, «Bona (Jean)», Dictionnaire de spiritualité, col. 1765.

15. Voir ibid., col. 1766 
cette théologie d'avoir un langage adapté à son objet, contre les «quelques personnes» qui «condamnent la théologie mystique, comme si elle contenait des termes obscurs, horribles, inouïs, inintelligibles, et différents de la doctrine des philosophes et des théologiens $»^{16}$. Pour Bona, «cette théologie mystique doit avoir ses termes, comme en ont tous les arts et toutes les sciences»: «puisqu'elle est entièrement surnaturelle, son principe, sa fin et ses moyens et ses voies pour tendre à la fin et pareillement ses termes et ses expressions surpassent l'ordre et les forces de la nature, et le langage de la sagesse humaine. $\gg^{17}$ Dans le traité Via compendii ad Deum, l'apologie porte moins sur le vocabulaire mystique que sur la distinction entre une science spéculative et une science pratique. Bona distingue «deux voies pour aller à Dieu», la voie «scolastique et commune» et la voie «mystique et secrète»:

La première demande beaucoup d'étude et de recherche, et n'est propre que pour des gens d'une profonde littérature: la seconde se peut acquérir partout, en tout temps, et par tout le monde. L'une est spéculative, et l'autre est pratique. Celle-là proprement est dans l'esprit, et celle-ci dans la volonté ${ }^{18}$.

Sous la plume de Bona, la voie mystique apparaît comme supérieure. Elle est plus sûre: «Celle-là [la scolastique] a sujet de craindre, parce qu'elle est exposée à l'illusion, à l'orgueil, et à la curiosité; celle-ci [la mystique] marche simplement et sûrement, parce qu'elle ne cherche que Dieu. $\gg^{19}$ Elle est plus facile à suivre: «Celle-là est longue, laborieuse et difficile: celle-ci est courte, prompte et très aisée. $\gg^{20}$ Elle mène plus loin dans la connaissance de Dieu: "Celle-là est bornée, et ne passe pas les limites des connaissances humaines: celle-ci étend le cœur à une infinité de choses, et s'élève au-dessus de toutes les connaissances naturelles. $»^{21}$ Toutefois, Bona ne considère pas comme inutile la voie scolastique. Il renvoie d'ailleurs explicitement à l'ouvrage qu'il lui a consacré, le traité Manuductio ad Coelum ${ }^{22}$.

16. G. Bona, Traité du discernement des esprits, p. 535.

17. Ibid.

18. G. Bona, Voie abrégée pour aller à Dieu, p. 4.

19. Ibid., p. 4-5.

20. Ibid., p. 5.

21. Ibid.

22. Voir la note 5 , p. 8, de S. Stroppa à son édition de la Via compendii ad Deum. Le traité Manuductio ad Cœelum paraît pour la première fois à Rome, chez 
La théologie mystique est donc définie par rapport à la théologie scolastique. Comme ses contemporains, Bona hérite d'une conception de la théologie comme science autonome, ayant son objet, sa méthode, ses lieux et ses institutions propres - une conception née avec l'émergence de la théologie des écoles aux XII ${ }^{\mathrm{e}}$ et $\mathrm{XIII}^{\mathrm{e}}$ siècles $^{23}$. C'est selon ce modèle que Bona cherche à établir la mystique comme une théologie à part entière, supérieure même à la scolastique. Alors que dans le traité De discretione spirituum, il semblait faire de la théologie mystique un art et une science, Bona indique, dans le traité Via compendii ad Deum, qu'elle «n'est pas à proprement parler une science, en tant que la science est une habitude acquise $»^{24}$. La précision est d'importance. Bona ne nie pas la prétention de la mystique à être une théo-logie, bien au contraire. Mais à cause de son objet propre, elle se situe au-delà des « sciences humaines [qui] s'apprennent par le moyen de l'imagination $»^{25}$, comme «la théologie scolastique [qui] apprend à l'homme à bien user des choses intelligibles, et la symbolique à se bien servir des sensibles $»^{26}$. La théologie mystique, elle, «est purement surnaturelle tant dans sa substance, que dans sa manière de procéder $»^{27}$. C'est pourquoi elle n'est pas une science acquise, mais un don de Dieu, «qui s'appelle Sagesse, mais dans son plus haut degré » ${ }^{28}$.

\section{UNE THÉOLOGIE PUREMENT SURNATURELLE}

Malgré la distinction qu'il pose entre les deux théologies, c'est sur un axiome des scolastiques que Bona fonde sa définition de la théologie mystique: «La disposition à recevoir une forme, doit être de même ordre que la forme même à laquelle elle dispose. $»^{29}$

\footnotetext{
A. Bernabò, en 1658. Il connaît de très nombreuses rééditions avant et après la mort de Bona, ainsi que quatre traductions en français entre 1665 et 1690.

23. Sur le passage de la «sacra doctrina» à la théologie comme science, voir Marie-Dominique Chenu, La Théologie comme science au XIII siècle, Paris, Vrin, 1927. Du même auteur, voir également La Théologie au XII siècle, Paris, Vrin, 1957.

24. G. Bona, Voie abrégée pour aller à Dieu, p. 17.

25. Ibid., p. 19.

26. Ibid., p. 18

27. Ibid., p. 19

28. Ibid., p. 18

29. Ibid., p. 24
} 
Or, comme «la théologie mystique est entièrement surnaturelle, il s'ensuit clairement qu'elle présuppose une manière d'état surnaturel qui lui soit proportionné. $»^{30}$ La doctrine des aspirations vise précisément à décrire ce passage d'un état où l'homme s'élève vers Dieu par ses qualités naturelles, avec l'aide de la grâce, à un état qui n'est plus que surnaturel. En arrière-plan du syllogisme présenté par Bona, on devine l'importance de la doctrine de la pure nature pour la question mystique. L'élaboration d'un système de la pure nature par les théologiens à partir du $\mathrm{XVI}^{\mathrm{e}}$ siècle ${ }^{31} \mathrm{a}$ eu des conséquences décisives et encore trop peu étudiées sur la manière dont les mystiques pensaient le processus d'union à Dieu. Intégrant le concept de pure nature, les doctrines mystiques développaient l'expression purement surnaturelle. À l'ordre naturel se surajoutant l'ordre surnaturel, il leur fallait penser le passage de l'un à l'autre. Cela supposait de discerner la part de la nature et la part de la grâce dans la vie de l'âme, comme on peut le voir, par exemple, sous la plume du jésuite Louis Lallemant:

Les saints anges n'ont jamais fait de ces actions que nous appelons de pure nature; ils renoncèrent pour jamais à leur amour propre, par le pur motif de l'amour de Dieu [...].

Nous devrions imiter cette fidélité des anges, agissant toujours par des principes surnaturels. Mais nous sommes tout plongés dans la nature, et la plupart de nos actions sont, ou purement naturelles, ou mêlées partie de la grâce et de la nature. Nous n'en faisons presque point qui soient toutes de la grâce et parfaitement surnaturelles ${ }^{32}$.

Dans son introduction à la théologie mystique, Bona se fonde lui aussi sur une telle distinction. Lorsqu'il traite de la contemplation, il précise qu'il entend parler «du passage de la manière d'agir humaine et naturelle, à celle qui est divine et surnaturelle $»^{33}$. Il doit ici bien plus à la scolastique moderne qu'à la scolastique médiévale. Notons toutefois que cette manière nouvelle de penser la relation entre nature et grâce sur la question de l'union mystique prolongeait probablement, et de façon inattendue, les débats médiévaux sur les causes. Dans une étude aussi magistrale que décisive, Jacob

30. Ibid.

31. Voir Henri de Lubac, Augustinisme et théologie moderne [1965], Paris, Cerf, 2008.

32. Louis Lallemant, Doctrine spirituelle [1694, posthume], éd. Dominique Salin, Paris, Desclée de Brouwer («Christus»), 2011, p. 133.

33. G. Bona, Voie abrégée pour aller à Dieu, p. 138. 
Schmutz a montré que l'évolution doctrinale sur la relation entre cause première et cause seconde avait permis l'élaboration d'une théologie de la nature pure à l'époque moderne ${ }^{34}$. Une enquête mériterait d'être menée sur le rôle qu'ont joué les doctrines médiévales des causes dans la mystique moderne. En pensant l'union à Dieu comme le passage d'un agir naturel sous l'action de la grâce à un agir purement surnaturel, les mystiques intègrent deux modèles de cause: il s'agit de passer d'un système de causes partielles concourantes (l'agir de Dieu et l'agir de l'homme sont chacun une partie de la cause unique de leur action commune) à un système de cause unique (Dieu est la seule cause de leur union, ce qui suppose un anéantissement de l'homme, ou du moins de son action). On le voit dans le traité Vita compendii ad Deum, lorsque Bona pense le passage de la méditation aux aspirations ${ }^{35}$, puis à la contemplation ${ }^{36}$. La méditation demande la mise en œuvre de facultés naturelles, telles que l'entendement et l'imagination. Les aspirations sont une élévation de l'homme vers Dieu qui suppose leur conspiration: «On les appelle inspirations, parce que nous aspirons à Dieu par elles, et qu'à travers elles, Dieu seconde nos efforts; assurément, tandis que nous aspirons à Dieu, nous ne soupirons qu' après Dieu. ${ }^{37}$ Des aspirations à la contemplation, l'accession à la théologie mystique est le "passage de la manière d'agir humaine et naturelle, à celle qui est divine et surnaturelle $»^{38}$. Il est frappant que la doctrine de Bona n'accorde plus de place à une conception des causes seconde (agir humain) et première (agir divin) comme deux causes totales subordonnées l'une à l'autre, conception qui était encore celle du thomisme traditionnel ${ }^{39}$. La défense de la théologie mystique comme une sagesse purement surnaturelle est caractéristique de l'occultation, dans le catholicisme moderne, de l'antique intuition de la théandrie: Dieu et l'homme font chacun la totalité de leur œuvre commune, parce que le Christ est vrai homme

34. Voir Jacob Schmutz, «La doctrine médiévale des causes et la théologie de la nature pure (XIII-XVII ${ }^{\mathrm{e}}$ siècles)», Surnaturel. Une controverse au cœur du thomisme du XX siècle, Revue thomiste, $\mathrm{n}^{\circ}$ 101/1-2, 2001, p. 217-264.

35. Voir G. Bona, Voie Abrégée pour aller à Dieu, p. 69-70 notamment.

36. Voir ibid., ch. 9.

37. «Quia illis aspiramus ad Deum, et per eas aspirat Deus conatibus nostris: dum vero ad Deum aspiramus, nil nisi Deum spiramus ». Ibid., p. 48.

38. Ibid., p. 138.

39. Voir l'article cité de J. Schmutz, p. 251. 
et vrai $\mathrm{Dieu}^{40}$. L'absence presque totale du Christ dans le discours de Bona sur la théologie mystique n'est pas le moindre signe de la séparation qu'opère le catholicisme classique entre la christologie et une pensée de l'union des volontés de l'homme et de Dieu. On mesure l'écart de Bona avec son maître en théologie mystique, Bernard, qui pouvait affirmer, en s'appuyant sur le vocabulaire du concile de Chalcédoine:

Ce n'est pas en partie la grâce, en partie le libre arbitre, mais l'une et l'autre qui accomplissent tout par une seule ouvre indivisible: certes, il accomplit tout et elle accomplit tout; mais de même qu'elle accomplit tout en lui, il accomplit tout par elle ${ }^{41}$.

\section{CirCularité de L'AMOUR ET PROGRÈS SPIRITUEL}

Le lecteur au fait des écrits spirituels du Moyen Âge aura reconnu dans la triade méditation / aspirations (c'est-à-dire oraison) / contemplation les trois degrés qui suivent la lecture dans L'échelle du cloître. Composé au XII ${ }^{\mathrm{e}}$ siècle, ce livre de Guigues II le chartreux a une importance considérable dans la réflexion sur la prière au XVII ${ }^{\mathrm{e}}$ siècle $^{42}$. En effet, cette description des étapes de la lectio divina comme une ascension vers Dieu sera une autorité sans cesse invoquée dans les débats sur les méthodes d'oraison. Notamment, défenseurs et contempteurs de l'oraison méthodique se réfèrent à lui, ce qui suppose, on s'en doute, de l'interpréter avec des catégories modernes. Bien qu'il ne soit pas cité, il est sans doute la source médiévale la plus importante dans le traité Via compendii ad Deum, car il donne en quelque sorte la matrice de la voie mystique décrite par Bona.

Pour comprendre le projet du feuillant, il faut prendre en compte son inscription dans la tradition d'une spiritualité scalaire, dont les

40. Voir S. Icard, Le Mystère théandrique. Action de Dieu et action de l'homme dans l'œuvre du salut, Paris, Honoré Champion, à paraître en 2014.

41. «Non partim gratia, partim liberum arbitrium, sed totum singula opere individuo peragunt: totum quidem hoc, et totum illa, sed ut totum in illo, sic totum ex illa». Bernard de Clairvaux, De gratia et libero arbitrio, $\S 47$. Sur la présence du vocabulaire chalcédonien dans le propos de Bernard, voir la fin du ch. 1 de mon essai Le Mystère théandrique.

42. Voir Ch. Belin, op. cit., p. 75-77; et S. Icard, Port-Royal et saint Bernard de Clairvaux, p. 309-327. Au XVII ${ }^{\mathrm{e}}$ siècle, l'ouvrage est fréquemment attribué à Bernard, surtout avant les années 1640. 
moines du $\mathrm{XII}^{\mathrm{e}}$ siècle ont été de si puissant relais. Certes, la voie mystique est présentée comme plus facile et plus courte pour aller à Dieu que la voie scolastique. Elle «se peut acquérir partout, en tout temps, et par tout le monde $»^{43}$. Mais elle reste une voie méthodique. À propos des aspirations, qui sont l'objet principal du livre, Bona précise que «cet exercice convient surtout à ceux qui ont déjà purifié leur âme des vices, et fait quelque progrès dans la voie illuminative, dans l'étude des vertus et la pratique de la méditation. Car après que l'âme s'est détachée de l'affection du siècle par le moyen de l'oraison mentale, de sorte qu'elle commence à sentir que le feu de l'amour divin est allumé en elle, laissant la méditation, elle passe à l'état des aspirations et de là enfin à la contemplation et à la théologie mystique. $\gg^{44}$ La pratique des aspirations n'est donc pas, pour Bona, une alternative aux prières méthodiques. Elle est une étape dans la progression vers l'union mystique. Après avoir indiqué, en suivant Jean de la Croix, les signes permettant de discerner qu'il est temps de passer de la pratique de la méditation à celle des aspirations ${ }^{45}$, Bona considère que cette dernière dure un an tout au plus ${ }^{46}$. En décrivant l'itinéraire qui mène à l'union mystique, il reprend avec des catégories modernes la réflexion des moines médiévaux sur les degrés de l'ascension vers Dieu.

Dans L'échelle du cloître, les quatre degrés correspondent à quatre états. La lecture est l'œuvre des commençants, la méditation celle des progressants, l'oraison celle des parfaits et la contemplation celle des bienheureux, état que l'on peut connaître par anticipation, mais pas de manière permanente durant cette vie ${ }^{47}$. Bona reprend cette correspondance, mais en la décalant d'un degré : la méditation est l'œuvre des commençants, les aspirations celle des progressants et la contemplation celle des parfaits. De la lecture, il n'est pas question dans son traité. Du fait de ce décalage, la perspective eschatologique est nettement moins marquée que dans le livre de Guigues. Il s'agit là d'un changement avec la conception des degrés développée dans la littérature monastique du $\mathrm{XII}^{\mathrm{e}}$ siècle: l'ultime degré de leurs échelles n'est atteint que dans la Jérusalem céleste;

43. G. Bona, Voie abrégée pour aller à Dieu, p. 4.

44. Ibid., p. 68

45. Voir ibid., p. 69-70.

46. Voir ibid., p. 99.

47. Voir Guigues II le chartreux, L'échelle du cloître, fin du ch. 6. 
c'est un degré in patria, que le viator ne peut connaître que de manière fugace et par anticipation. Tout comme l'occultation de la christologie, l'atténuation de cette tension eschatologique n'est pas anodine. Alors que Guigues insiste sur la nécessité de descendre souvent d'un degré à cause de la faiblesse humaine, sans pour autant perdre le désir de voir Dieu, Bona présente l'itinéraire de telle sorte que le passage d'un degré à l'autre suppose une certaine rupture. Comme nombre de ses contemporains, le feuillant conçoit les degrés comme des ordres. Dans la littérature monastique du $\mathrm{XII}^{\mathrm{e}}$ siècle, l'ascension des degrés est une assomption: le degré inférieur est non seulement dépassé, mais assumé dans le degré supérieur. Pour un moderne comme Bona, l'ascension des degrés implique une rupture: le degré inférieur est certes proportionné à l'ordre supérieur, mais ne s'accomplit pas en luii ${ }^{48}$.

Cette substitution est liée à l'occultation de l'intuition théandrique. Dans L'échelle du cloître, la question de la part respective que Dieu et l'homme ont dans leur œuvre commune ne se pose pas. Chaque degré est intégralement œuvre de l'un et de l'autre. Chez Bona, qui n'est en cela pas du tout original à son époque, l'ascension par degré suppose de penser le moment où Dieu assumera seul l'œuvre commencée par la nature humaine mue par la grâce - le passage d'un acte naturel à un acte purement surnaturel. On retrouve exactement le même type de préoccupation dans les lectures contradictoires de L'échelle du cloître qui sont faites à Port-Royal ${ }^{49}$. Pour les modernes, réfléchir sur l'oraison, c'est penser le passage d'une activité humaine à une activité divine, discerner avec autant d'assurance que possible l'œuvre invisible de la grâce, la «surnaturalité» de l'acte, pour reprendre un terme de Pierre Nicole ${ }^{50}$. Dans le traité Via compendii ad Deum, cette transformation de la doctrine médiévale des degrés tient sans doute à une superposition opérée par Bona. Aux trois états distingués par la tradition monastique (commençants, progressants, parfaits) correspondent les trois voies distinguées par Denys l'Aéropagite (voie purgative, voie illuminative, voie unitive), conçues ici comme

48. Voir S. Icard, Port-Royal et saint Bernard de Clairvaux, p. 487-488.

49. Voir ibid., p. 309-327.

50. Pierre Nicole, Réfutation des principales erreurs des quiétistes, Paris, É. Josset, 1695, p. 100. 
trois ordres hiérarchiques. La voie abrégée vers Dieu est pensée comme le passage d'une voie à une autre ${ }^{51}$.

Malgré sa conceptualisation moderne des degrés de l'ascension mystique, Bona retrouve une intuition chère aux moines du $\mathrm{XII}^{\mathrm{e}}$ siècle: le degré supérieur récapitule ce qui est donné au degré inférieur. Certes, il ne s'agit pas ici de l'accomplissement du degré inférieur dans le degré supérieur, mais d'une correspondance et même d'une communication entre les trois voies, chacune d'entre elles développant, selon les besoins propres à chaque état, les caractéristiques des deux autres:

Encore que nous ayons distingué les trois états dont nous venons de parler, selon les trois exercices, de la pureté, de la lumière, et de l'amour, il ne le faut pas prendre néanmoins comme si la purgation de l'âme ne s'étendait point aux autres états, ni l'illumination au premier ou au troisième, ni la perfection aux deux précédents: car tous les actes hiérarchiques se rencontrent dans chacun de ces états; en sorte, par exemple, que non seulement la purgation des péchés, mais encore la connaissance de la vérité, et l'amour de Dieu ont lieu dans la voie purgative, et ainsi des autres; et ces actes se font d'autant plus excellemment, que l'état où on les fait est plus sublime. Toutefois chaque exercice est destiné à chaque état, parce qu'il lui est propre et essentiel, et qu'on doit rapporter les deux autres exercices à celui de l'état où l'on est: comme dans le premier état, par exemple, il faut rapporter la lumière et l'amour à la purgation de l'âme; dans le second, la purgation et l'amour à l'illumination, dans le troisième, la pureté et la lumière à l'amour. Ainsi, la communication de ces actes fait que la douleur que l'on a de ses péchés, est adoucie par l'amour; et que l'amour accompagné de la considération des sujets d'une juste douleur, devient plus sûr et plus fervent: et tandis qu'on mêle les actes et les exercices d'une voie avec les actes et les exercices de l'autre, les commençants aspirent sans cesse à un état plus élevé; et les avancés et les parfaits conservent le souvenir et le sentiment

51. «Dans le premier état, qui est celui de la voie purgative, sont les pécheurs, qui après leur conversion punissent leurs péchés passés, et combattent pour n'y plus retomber; coupent les racines de leurs mauvaises habitudes et font mourir leurs passions. C'est dans ce dessein qu'ils châtient leur chair par les jeûnes, par les veilles, par les travaux et par d'autres austérités et qu'ils s'adonnent aux larmes et à la contrition. Dans le second état, qui est celui des avancés, sont ceux qui se dépouillant de l'affection des choses terrestres, s'appliquent à la pureté, et à l'acquisition des vertus: car la vertu est la propre et véritable lumière de l'âme. C'est pour cela qu'ils s'animent par de fréquentes méditations sur les vertus et sur la doctrine de Jésus-Christ, et qu'ils s'enflamment à l'imiter. Enfin, ceux-là se reposent dans le troisième état, qui ayant déjà purifié leur entendement, méritent d'être unis à Dieu par des nœuds secrets, et jouissent d'une paix qui surpasse tout sentiment». G. Bona, Voie abrégée par aller vers Dieu, p. 90-91. 
des choses qui sont les fondements de la perfection et les premiers principes de la piétét ${ }^{52}$.

Cette communication des actes hiérarchiques répond probablement à ce qui pourrait de prime abord apparaître comme une tension dans le traité Vita compendii ad Deum. Bona conçoit la voie mystique comme une ascension par degré, dans le sens que nous avons cherché à établir, mais aussi comme une sorte de circularité. À plusieurs reprises, Bona montre que la voie mystique commence et s'achève par l'amour: "Par un cercle admirable commençant par l'amour, [elle] s'avance vers l'amour et finit par l'amour par des mouvements affectueux et des aspirations enflammées. $\gg^{53}$ Comment penser une voie qui soit à la fois progressive et circulaire? Il ne semble pas que Bona pense cette ascension comme une spirale - une image qui pourrait pourtant décrire l'idée si présente dans la tradition monastique d'une progressive intériorisation, par un retour cyclique, de ce qui est donné dès l'origine ${ }^{54}$. Si l'on voulait tenter une image, on pourrait dire que Bona pense la voie mystique comme des disques superposés les uns aux autres, composés de cercles concentriques, eux-mêmes disposés selon l'ordre propre à chaque degré (ce qui est concentrique à un degré devenant plus ou moins périphérique dans les autres). Passer d'un degré à l'autre suppose donc une rupture, mais ce qui était donné au degré inférieur est repris au degré supérieur selon son ordre propre. À chaque niveau, c'est le même amour qui se trouve à l'origine et à la fin. Bona l'indique explicitement à propos des trois états : «Il n'y a qu'une charité à laquelle ils aspirent tous, mais les degrés et les accroissements en sont divers. $\gg^{55}$

On aurait tort de ne voir en Bona qu'un simple compilateur des autorités spirituelles jalonnant l'histoire de la mystique chrétienne. Il lit les auteurs anciens avec des grilles de lecture qui sont celles de son époque. Son inscription revendiquée dans la tradition conduit à une synthèse moderne sur la voie mystique. Le traité Via compendii ad Deum, bien qu'il se présente comme la description

52. Ibid., p. 94-95.

53. Ibid., p. 5-6.

54. Voir S. Icard, Le Mystère théandrique, ch. 3.

55. G. Bona, Voie abrégée pour aller à Dieu, p. 91. 
d'un continuum doctrinal sur la question mystique, montre, s'il en était besoin, qu'il n'est pas de retour aux sources sans une conceptualisation implicite, que seul le recul de l'histoire permet de mettre en lumière. Une simple liste des autorités mobilisées par Bona dans son traité montre qu'en matière de mystique, l'héritage médiéval est au moins aussi important que celui des Pères de l'Église. Le Moyen Âge n'apparaît pas comme un simple relais de l'âge patristique, pour lequel le catholicisme classique avait une dévotion immense. Sa position médiane entre les Pères et les modernes lui donne un rôle prépondérant dans l'élaboration des concepts qui fondent les lectures que le $\mathrm{XVII}^{\mathrm{e}}$ siècle a pu faire de la tradition chrétienne. Pour étudier l'histoire des idées religieuses, il est donc nécessaire de surmonter l'opposition canonique du Moyen Âge et des Temps modernes.

icard.simon@orange.fr 